\title{
Consenso chileno SER-SOCHINEP para el manejo del asma severo en el niño ${ }^{\#}$
}

\author{
ANA MARÍA HERRERA G.* (Coordinadora), SELIM ABARA E.**, CECILIA ÁLVAREZ G.***, \\ CLAUDIA ASTUDILLO M.****, RAÚL CORRALES V.***, EVELYN CHALA C.*****, \\ TATIANA ESPINOZA P.******, ANDRÉS KOPPMANN A.********, VIVIANA LEZANA S.*******, \\ ROSSANA MAMANI J.*, MARÍA ANGÉLICA PÉREZ H.* y CRISTINA PIERRY V.**
}

\section{Chilean consensus on management of severe asthma in children}

Severe asthma represents less than 5\% of all asthma, but these patients are responsible for a disproportionate share of health care costs, morbidity and mortality associated with the disease. Severe problematic asthma corresponds to patients that fail to achieve asthma control despite being prescribed maximal therapies. This group is divided into severe difficult to control asthma and severe therapy resistant asthma. It is important to note this division as it leads to important differences in medical prognosis and therapy. Difficult to control asthma is the term used to describe children with significant ongoing symptoms that are due to underlying modifiable factors that, when addressed, lead to better control without increasing therapy intensity. On the other hand, therapy resistant asthma does not present modifiable factors, thus other treatments, including biological therapies, are justified.

Key words: Severe asthma, refractory asthma, asthma control.

\section{Resumen}

Se estima que el asma severo tiene una prevalencia menor al 5\%, sin embargo se asocia a una alta morbimortalidad, dando cuenta de alrededor de $50 \%$ de los gastos en salud por esta enfermedad. El término "asma severo problemático" se aplica a los pacientes asmáticos que no alcanzan el control de la enfermedad a pesar de recibir la máxima terapia convencional disponible. Este grupo se subdivide en asma severa de difícil control y asma severa resistente a tratamiento. La diferenciación de ambos grupos es fundamental ya que tiene implicancias terapéuticas y pronósticas. En el asma de dificil control existen factores potencialmente modificables que al ser corregidos permiten el control de la enfermedad, sin la necesidad de escalar en la terapia. Por otra parte, en el asma resistente a tratamiento no existen factores modificables, por lo que en este grupo está justificado el uso de otras terapias incluidas las biológicas.

Palabras clave: Asma severa, asma de dificil control, asma resistente a tratamiento.

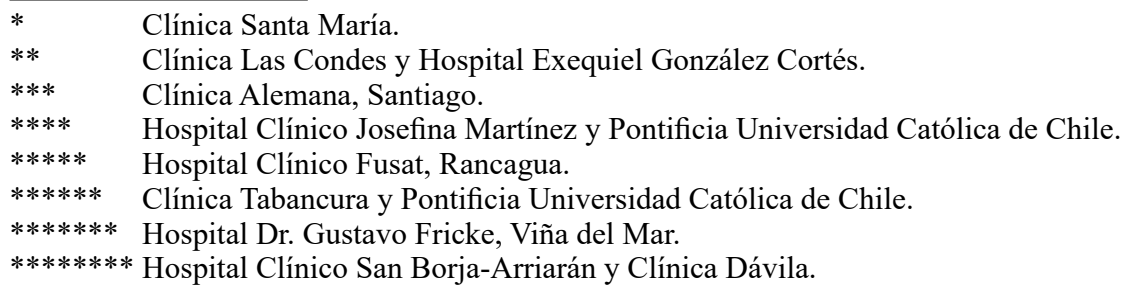

"Documento elaborado por las Comisiones de Asma Pediátrico de la Sociedad Chilena de Enfermedades Respiratorias (SER) y Sociedad Chilena de Neumología Pediátrica (SOCHINEP).

Considerando la importancia de este tema, la Sociedad Chilena de Enfermedades Respiratorias y la Sociedad Chilena de Neumología Pediátrica han acordado publicar este consenso conjuntamente en sus órganos oficiales Revista Chilena de Enfermedades Respiratorias y Revista Neumología Pediátrica.

Declaración de conflicto de intereses: Los Drs. Selim Abara E., Cecilia Álvarez G., Claudia Astudillo M., Raúl Corrales V., Evelyn Chala C., Tatiana Espinoza P., Andrés Koppmann A., Viviana Lezana S., Rossana Mamani J., María Angélica Pérez H, y Cristina Pierry V. declaran no presentar conflictos de intereses. La Dra. Ana María Herrera G., recibió en 2015 un Grant del Laboratorio Novartis para un proyecto de investigación, prospectivo y descriptivo y no intervencional acerca de asma en niños. 


\section{Introducción}

El asma bronquial es la enfermedad crónica más prevalente en la infancia, consume gran cantidad de recursos en salud y afecta la calidad de vida del paciente y su familia ${ }^{1,2}$. La gran mayoría de los pacientes logra ser tratado adecuadamente con dosis bajas y seguras de corticoides inhalados. Sin embargo, existe un grupo cercano al 5\% que no logra el control de la enfermedad a pesar de recibir altas dosis de corticoides inhalados asociados a agonistas $\beta$-2 adrenérgicos de acción prolongada (LABA), antileucotrienos e incluso corticoides orales. Este tipo de pacientes, a pesar de su baja prevalencia, presenta una altísima morbilidad asociada, dando cuenta de casi $50 \%$ de los gastos totales en salud relacionados con la enfermedad ${ }^{2-4}$.

\section{Definiciones}

De acuerdo a las guías GINA la severidad del asma se define en base a la intensidad del tratamiento requerido para alcanzar un buen control ${ }^{5}$. El control a su vez se refiere al grado en que las manifestaciones de asma pueden observarse o han sido reducidas o eliminadas por el tratamiento ${ }^{5}$. El control del asma incluye dos componentes que siempre deben ser evaluados: el control de los síntomas y el riesgo futuro de eventos adversos $^{5}$. De acuerdo a GINA el asma severo es aquel que requiere tratamiento en etapa 4 para alcanzar el control de la enfermedad o aquel que a pesar de estar en esta etapa no lo logra ${ }^{5}$. La guía internacional de asma severo, elaborada por la Sociedad Europea de Enfermedades Respiratorias (ERS) junto con la Sociedad Americana de Tórax (ATS), coincide con la definición de asma severo de GINA, pero agrega que el paciente debe mantenerse en este nivel de tratamiento en los últimos 12 meses $^{6}$. Además se incluye en la categoría de asma severo a los pacientes que han utilizado corticoides sistémicos por 6 meses o más durante el año previo ${ }^{6}$. En este documento además se establece claramente lo que se considera asma no controlado (Tabla 1).

De acuerdo a la Organización Mundial de la Salud el asma severo incluye tres grupos: asma severo no tratado, asma severo difícil de tratar o de difícil control y asma severo resistente a tratamiento ${ }^{4}$.

\section{Asma severo no tratado}

Se refiere a los pacientes asmáticos que no están recibiendo tratamiento por falta de diagnóstico o por falta de disponibilidad o acceso al tratamiento. Esto último se puede observar especialmente en países del tercer mundo ${ }^{6,7}$.

\section{Asma severo difícil de tratar o de difícil control}

Se refiere a pacientes con un pobre control de la enfermedad por alguna de las siguientes causas: error en el diagnóstico, comorbilidades no tratadas, falta de adherencia al tratamiento, deficiente técnica inhalatoria $\mathrm{y} / \mathrm{o}$ factores ambientales (exposición a alérgenos, irritantes y humo de cigarrillo). En este grupo de pacientes, el manejo adecuado de cada uno de estos factores permite alcanzar el control de la enfermedad ${ }^{3,4,6,7}$. La falta de adherencia al tratamiento es por lejos el más importante de los factores mencionados.

\section{Asma severo resistente a tratamiento o refractario}

Se refiere a pacientes asmáticos cuya enfermedad no se logra controlar a pesar de un manejo óptimo, que incluye el tener bajo control todos los factores mencionados en el asma difícil de tratar, asociado al hecho de estar recibiendo la terapia de mayor intensidad disponible $e^{3,6,7,8,9}$.

\section{Asma severo problemático}

Es un término que se utiliza para englobar a los pacientes asmáticos que no responden a la terapia indicada, con presencia de síntomas crónicos, exacerbaciones agudas severas y/o limitación persistente al flujo aéreo ${ }^{7}$. Este término incluye al asma severa difícil de tratar o de difícil control y al asma severo resistente a tratamiento $0^{6,7,10}$.

\section{Epidemiología}

El asma severa representa aproximadamente el $5 \%$ de los casos de asma en la infancia ${ }^{3}$. De acuerdo a la literatura internacional, el 39 a 55\%

\section{Tabla 1. Asma no controlado ${ }^{6}$}

Asma no controlado se define por la presencia de al menos uno de los siguientes criterios:

1) Pobre control de síntomas: ACQ > 1,5, ACT $<20$ (o "no bien controlado" de acuerdo a las guías NAEPP/ GINA)

2) Exacerbaciones severas frecuentes: 2 o más curas de corticoides sistémicos ( $>3$ días cada una) durante los últimos 12 meses

3) Exacerbaciones graves: al menos 1 hospitalización, requerimiento de UTI o ventilación mecánica durante el año previo

4) Limitación al flujo aéreo: mantiene $\mathrm{VEF}_{1}<80 \%$ del predicho después de dosis adecuadas de broncodilatador 
del total de niños con asma severo problemático corresponde al grupo de difícil control ${ }^{11-13}$. La identificación de este subgrupo de pacientes es de gran importancia ya que al tratar los factores que inciden negativamente en la evolución de la enfermedad se logra el control, sin necesidad de progresar en el estudio ni escalar en la terapia ${ }^{11,12}$. Al comparar a los pacientes portadores de asma severo difícil de tratar o de difícil control con aquellos que presentan asma severo resistente a tratamiento vemos que estos últimos tienen menor función pulmonar, mayor hiperreactividad bronquial, niveles más altos de $\mathrm{F}_{\mathrm{E}} \mathrm{NO}$ (fracción espirada de óxido nítrico), mayor sensibilización a aeroalergenos y mayor uso de corticoides orales en comparación con los primeros ${ }^{14}$.

$\mathrm{Al}$ igual que en el adulto, se ha establecido que el asma severo en el niño es una enfermedad heterogénea y que estaría compuesta por al menos 4 fenotipos diferentes: a) de inicio tardío con función pulmonar normal; b) de inicio temprano con función pulmonar normal; c) de inicio temprano con limitación obstructiva leve y d) de inicio temprano con limitación obstructiva avanzada ${ }^{6}$.

\section{Enfrentamiento del paciente con asma severo problemático}

Frente a un paciente con asma severo problemático lo primero que se debe hacer es evaluar si se trata de un paciente con asma severo de difícil control o si es severo resistente a tratamiento. Sólo cuando se han descartado las causas de asma de difícil control podemos decir que estamos frente a un paciente con asma resistente a tratamiento ${ }^{4,7,11}$.

Al enfrentar a un paciente con asma severo problemático se deben seguir los pasos que se detallan a continuación para poder distinguir entre ambos subgrupos.

\section{Confirmar el diagnóstico de asma}

Este primer paso es de gran importancia ya que es posible que el paciente no responda a la terapia simplemente porque no es asmático ${ }^{11,15}$. Para cumplir con este punto es fundamental una detallada anamnesis y examen físico tendientes a descartar otras patologías que pueden confundirse con asma bronquial (Tabla 2) ${ }^{15}$. Los exámenes a solicitar dependerán de la evaluación que se haga del paciente, pero esta Comisión recomienda como mínimo solicitar radiografía de tórax, test del sudor, hemograma y recuento de inmunoglobulinas.

Junto con un adecuado diagnóstico diferencial es importante confirmar el diagnóstico de asma bronquial con la historia clínica, el examen físico y algunos exámenes de laboratorio ${ }^{5}$. Los pacientes con asma tienen historia de episodios recurrentes de obstrucción bronquial caracterizados por sibilancias, tos, disnea y sensación de opresión torácica. Los síntomas son variables en intensidad y en frecuencia de presentación, em-

Tabla 2. Diagnóstico diferencial

\begin{tabular}{|c|c|}
\hline Enfermedades digestivas & $\begin{array}{l}\text { Aspiraciones recurrentes } \\
\text { Trastorno de deglución } \\
\text { Fístula traqueo-esofágica }\end{array}$ \\
\hline Enfermedades de la vía aérea alta & Disfunción de cuerda vocal \\
\hline Enfermedades obstructivas del árbol traqueo-bronquial & Cuerpo extraño \\
\hline Malformación de la vía aérea & $\begin{array}{l}\text { Anillos traqueales completos } \\
\text { Tráqueo-broncomalacia } \\
\text { Anillo vascular } \\
\text { Estenosis traqueal } \\
\text { Tumor endobronquial }\end{array}$ \\
\hline Enfermedades cardiovasculares & $\begin{array}{l}\text { Cardiopatía congénita } \\
\text { Insuficiencia cardíaca }\end{array}$ \\
\hline Enfermedades bronco-pulmonares & $\begin{array}{l}\text { Displasia broncopulmonar } \\
\text { Fibrosis quística } \\
\text { Disquinesia ciliar primaria } \\
\text { Bronquiectasias } \\
\text { Bronquiolitis obliterante }\end{array}$ \\
\hline Enfermedades infecciosas e inmunológicas & $\begin{array}{l}\text { Inmunodeficiencias } \\
\text { Aspergilosis broncopulmonar alérgica } \\
\text { Anafilaxia }\end{array}$ \\
\hline
\end{tabular}


peorando habitualmente en la noche. El principal desencadenante de las crisis de asma son las infecciones virales. En los períodos intercríticos los pacientes pueden tener síntomas como tos o sibilancias en relación a la exposición a alérgenos, ejercicio, aire frío, risa o llanto. Al examen físico se puede encontrar disminución del murmullo pulmonar y sibilancias de intensidad variable. La mejoría de los signos de obstrucción bronquial al examen físico con el uso de broncodilatador es de gran utilidad para confirmar clínicamente el diagnóstico de asma. La obstrucción bronquial también puede ser documentada mediante una espirometría, siendo de gran ayuda para el diagnóstico una respuesta broncodilatadora positiva. Cuando la espirometría resulta normal es importante demostrar la presencia de hiperreactividad bronquial con pruebas de provocación como el test de metacolina y test de ejercicio. También se debe certificar la naturaleza alérgica del asma mediante test cutáneo e IgE total ${ }^{5}$.

\section{Evaluar la presencia de comorbilidades}

La presencia de comorbilidades no tratadas adecuadamente contribuyen a un mal control del asma, por lo que siempre deben ser evaluadas. El diagnóstico y tratamiento oportuno de estas condiciones debe ser considerado en niños con asma de difícil control para asegurar un mejor pronóstico ${ }^{5}$.

\section{Rinitis alérgica}

Cerca de 60 a $80 \%$ de los pacientes con asma tienen concomitantemente rinitis alérgica, siendo los corticoides intranasales el tratamiento de elección para esta condición ${ }^{16,17}$. En adultos con asma se ha demostrado que el tratamiento de la rinitis se asocia a una disminución de la frecuencia de hospitalización y visitas al servicio de urgencia ${ }^{18}$. Aunque en la literatura no ha sido demostrada tal asociación en niños con asma, de todas maneras se aconseja el tratamiento de la rinitis alérgica en esta población.

\section{Reflujo gastroesofágico (RGE)}

La prevalencia de RGE en niños asmáticos es dos veces mayor que en la población general, sin embargo su evaluación y tratamiento sólo están indicados cuando los pacientes son sintomáticos ${ }^{19}$. No se recomienda el uso rutinario de inhibidores de la bomba de protones en pacientes con asma severo ${ }^{20}$.

\section{Obesidad}

La relación entre obesidad y asma es compleja y aun no completamente comprendida. Varios estudios demuestran una mayor prevalencia de asma en niños con sobrepeso, siendo esta asociación más marcada en mujeres ${ }^{21}$. El asma es más difícil de controlar en pacientes obesos, lo que puede reflejar un tipo diferente de inflamación de la vía aérea y comorbilidades asociadas al sobrepeso como apnea obstructiva del sueño y reflujo gastroesofágico. Se ha demostrado que una pérdida del 5 a $10 \%$ del peso corporal en adultos con asma y sobrepeso conduce a una mejoría del control de la enfermedad, de la calidad de vida, de la función pulmonar y a un menor uso de medicamentos ${ }^{22}$. Por lo anterior se debe calcular el índice de masa corporal en los pacientes con asma severo e indicar reducción de peso en aquellos con obesidad y sobrepeso ${ }^{5}$.

\section{Respiración disfuncional}

Se define como cambios crónicos o recurrentes en el patrón respiratorio, lo que causa síntomas diversos como disnea, dolor torácico, opresión torácica, suspiros, disnea inducida por ejercicio, bostezos frecuentes e hiperventilación. Se ha demostrado una mayor prevalencia de esta disfunción en adultos con asma. No existe un estándar de oro para el diagnóstico y no hay estudios al respecto en niños ${ }^{23}$. Se ha demostrado que una intervención breve con fisioterapia es capaz de mejorar la calidad de vida de estos pacientes ${ }^{24}$.

\section{Alergia alimentaria y anafilaxis}

La incidencia de alergia alimentaria en la población general de niños menores de 3 años es de $8 \%$, sin variar en la población asmática. La alergia alimentaria y el asma son enfermedades atópicas que pueden coexistir, sin embargo es muy raro y atípico que los síntomas de asma sean la única manifestación de alergia alimentaria ${ }^{25}$. El antecedente de anafilaxis alimentaria confirmada en un paciente con asma, se considera un factor de riesgo para presentar exacerbaciones graves, por lo que estos pacientes deben ser entrenados en el uso de autoinyectores de adrenalina ${ }^{26}$.

\section{Trastornos psiquiátricos}

La presencia de depresión, ataques de pánico $\mathrm{y}$ trastornos de ansiedad son más prevalentes en adolescentes con asma y se asocian con mal control de la enfermedad, deficiente adherencia al tratamiento, peor calidad de vida y aumento de las exacerbaciones y visitas al servicio de urgencia ${ }^{25}$. Síntomas depresivos son un factor de riesgo identificado en niños y adolescentes que mueren por asma. Por otro lado, los trastornos de ansiedad pueden simular exacerbaciones de asma. Lamentablemente aunque se reconozcan 
los trastornos mentales en niños asmáticos, sólo 1 de 5 recibe el tratamiento farmacológico y psicoterapéutico adecuado ${ }^{27}$.

\section{Asegurar que las bases del tratamiento estén siendo cumplidas}

La correcta evaluación de este punto es fundamental antes de catalogar como severo a un paciente con asma bronquial.

\section{Adherencia}

La adherencia al tratamiento se refiere al grado en que el comportamiento del paciente se corresponde con las recomendaciones acordadas con el médico. La no adherencia al tratamiento es la principal causa de mal control en los pacientes asmáticos $^{28}$. Se ha demostrado que la adherencia a los medicamentos controladores debe superar el $75 \%$ de lo indicado para que el control del asma sea satisfactorio ${ }^{29,30}$. Desgraciadamente se ha visto que los niveles de adherencia en pacientes asmáticos oscilan entre el 30 y $50 \%$ de las dosis $\operatorname{prescritas}^{11,29}$. La deficiente adherencia al tratamiento se asocia a falta de control de la enfermedad y aumento del riesgo de exacerbaciones. La falta de adherencia al tratamiento puede ser accidental o deliberada. La falta de adherencia accidental o no intencional puede ocurrir cuando hay problemas de comprensión de las indicaciones por parte de padres y pacientes o debido al simple olvido. Por otro lado, la no adherencia deliberada o intencional se produce por rechazo al diagnóstico o al tratamiento. Los padres y pacientes no asumen la enfermedad como crónica y muchas veces sienten temor a potenciales efectos adversos de los medicamentos ${ }^{31}$. Esta última es la más difícil de superar. La adherencia no es fácil de objetivar y se han utilizado distintos métodos para medirla, tales como entrevistas o cuestionarios, medición de los medicamentos consumidos a través del registro de farmacia, peso de los inhaladores o a través del uso de dispositivos electrónicos de registro instalados en los inhaladores. Los métodos que evalúan cumplimiento por entrevistas o cuestionarios son altamente inexactos y tienden a sobrevalorar la adherencia cuando se los compara con métodos más objetivos como los dispositivos electrónicos ${ }^{32}$. Las estrategias para lograr mejorar la adherencia deben incluir aspectos educativos y cambios conductuales, lo que se verá con mayor detalle en la sección de manejo ${ }^{32,33}$.

\section{Técnica inhalatoria}

La vía inhalada es de elección para la administración de la mayoría de los fármacos empleados en el tratamiento del asma bronquial. El principal inconveniente de la inhaloterapia es la dificultad de los pacientes para utilizar correctamente los dispositivos generadores de aerosol. El uso adecuado de estos dispositivos es crucial ya que su empleo incorrecto puede ocasionar un control subóptimo de la enfermedad ${ }^{34}$. El éxito de la aerosolterapia depende de la correcta elección del dispositivo para cada paciente y del conocimiento de las técnicas de uso por parte de los padres y/o cuidadores, incluyendo personal de salud ${ }^{34,35}$. Los dispositivos utilizados para generar aerosoles son: inhaladores presurizados de dosis medida (IDM) que deben ser usados siempre junto a una cámara espaciadora con boquilla, nebulizadores e inhaladores de polvo seco (DPI) ${ }^{36}$. En la práctica clínica los IDM son los más usados y le siguen en frecuencia los DPI . La recomendación de las guías GINA para la elección del dispositivo se puede ver en la Tabla 3.

\section{Consideraciones generales para el uso de IDM más aerocámara $a^{34,35}$}

Es fundamental educar a los padres y/o cuidadores en la técnica inhalatoria correcta. Luego de haber recibido la información, los padres y/o cuidadores, deben demostrar el uso correcto, repitiendo todos los pasos de la maniobra de inhalación. Para la correcta entrega del medicamento siempre se debe usar aerocámara, de preferencia con válvula. Lo ideal es que la aerocámara tenga válvula inspiratoria y espiratoria. La inspiratoria permite el paso del aire desde la cámara hacia la vía aérea del niño y la espiratoria permite que exhale el aire sin volver a reintroducirlo en la cámara. Las partículas del aerosol sedimentan en las paredes de las cámaras plásticas como re-

\section{Tabla 3. Dispositivos disponibles ${ }^{5}$}

\begin{tabular}{|cll|}
\hline Edad & \multicolumn{1}{|c|}{ Dispositivo preferido } & \multicolumn{1}{c|}{ Dispositivo alternativo } \\
Niños mayores de 4 años & $\begin{array}{l}\text { Inhalador de cartucho presurizado + cámara } \\
\text { espaciadora con pieza bucal }\end{array}$ & $\begin{array}{l}\text { Inhalador de cartucho presurizado }+ \\
\text { cámara espaciadora con mascarilla facial } \\
\text { o nebulizador con pieza bucal o mascarilla } \\
\text { facial }\end{array}$ \\
\hline
\end{tabular}


sultado de las fuerzas electrostáticas. Es por eso que se recomienda el lavado una vez por semana con agua tibia y detergente, lo que disminuye las carga electrostática y aumenta significativamente el depósito pulmonar. Esto también se logra con el uso de aerocámaras metálicas. Al posicionar la aero-cámara se debe asegurar que los labios queden perfectamente sellados alrededor de la boquilla ya que una mínima separación reduce drásticamente la cantidad de fármaco inhalado (Tabla 4).

\section{Consideraciones generales para el uso de $D P I^{34,35}$}

Este tipo de inhalador precisa ser cargado para cada dosis contenida en el dispositivo. Su uso requiere que el paciente sea capaz de realizar un flujo inspiratorio suficiente para lograr un buen depósito pulmonar. Este varía entre 35 a $70 \mathrm{~L} /$ min según el tipo de dispositivo, lo que no siempre es posible lograr en escolares de menor edad (Tabla 5).

\section{Evaluar la exposición a factores ambientales}

\section{Alérgenos}

Se ha demostrado que la exposición persistente a alérgenos a los cuales el paciente está sensibilizado, tales como ácaros del polvo, caspa de gato, caspa de perro y cucaracha, aumentan los síntomas y las exacerbaciones de asma ${ }^{37}$. Lo anterior es especialmente relevante cuando además se asocia una infección viral ${ }^{38}$. En la literatura hay nueva evidencia científica acerca de la asociación causal entre la presencia de humedad en el domicilio y exacerbaciones de asma, lo cual no está limitado sólo a pacientes sensibilizados a polvo de habitación o a hongos ${ }^{37}$.

\section{Humo de cigarrillo}

Es la principal fuente de material particulado intradomiciliario $^{39}$. Se ha demostrado que la exposición crónica al humo del cigarrillo se asocia a un aumento de las exacerbaciones de asma y por otro lado la exposición pasiva o activa al humo de cigarrillo en pacientes con asma puede causar resistencia al tratamiento con corticoides inhalados ${ }^{37}$. De esta manera la exposición a este contaminante es un factor muy importante a evaluar en pacientes con asma severo problemático ${ }^{38}$. Hay que tener en consideración que la mayoría de las veces, el reporte de los padres acerca del tabaquismo intradomiciliario no es confiable, por lo que se recomienda la medición de cotinina en sangre, saliva u orina ${ }^{38}$.
Tabla 4. Técnica Inhalatoria de IDM + aerocámara en el niño $\geq$ de 6 años ${ }^{34-36}$

El paciente debe estar de pie o sentado

- Retirar la tapa del inhalador, agitar y conectar a la aerocámara

Situar la boquilla de la aerocámara entre los labios del niño (por detrás de los dientes)

- Espirar profundamente (afuera antes de ponerse la boquilla)

Apretar el pulsador una vez con la cámara horizontal

- Inspirar lenta y profundamente

- Realizar una pausa inspiratoria de 10 s y luego espirar lentamente

- Repetir los pasos para cada dosis con intervalos de 30 a $60 \mathrm{~s}$ entre dosis

Retirar el inhalador y taparlo

Enjuagar la boca con agua

Tabla 5. Técnica Inhalatoria para los dispositivos de polvo seco $^{34-36}$

- Cargar el dispositivo según las instrucciones que se especifican en cada producto

- Espirar profundamente con el inhalador alejado de la boca

Colocar la boquilla entre los labios

- Inspirar fuerte y sostenidamente

Retirar el inhalador de la boca, realizar una pausa de $10 \mathrm{~s}$ y espirar lentamente

- Cerrar el inhalador y enjuagar la boca al finalizar la inspiración

\section{Contaminantes}

Contaminantes intradomiciliarios: Los más importantes son los derivados de la combustión de madera, parafina y gas en estufas o cocinas. En el proceso de combustión se produce monóxido de carbono (CO), dióxido de azufre $\left(\mathrm{SO}_{2}\right)$, dióxido de nitrógeno $\left(\mathrm{NO}_{2}\right)$, material particulado con diámetro aerodinámico menor a $10 \mu \mathrm{m}\left(\mathrm{PM}_{10}\right) \mathrm{y}$ material particulado con diámetro aerodinámico menor a 2,5 $\mu \mathrm{m}\left(\mathrm{PM}_{2,5}\right)^{39}$. De todos los mencionados el más importante es el $\mathrm{NO}_{2}$, demostrándose asociación entre la exposición a este contaminante y exacerbaciones de asma ${ }^{37}$. De los sistemas de calefacción por combustión el más contaminante es la estufa a leña, seguido por parafina, siendo el menos contaminante el gas licuado ${ }^{40}$. 
Contaminantes extradomiciliarios: Son generados principalmente por el uso de combustibles fósiles en vehículos a motor y en procesos industriales $^{39}$. Los principales contaminantes extradomicilarios son $\mathrm{CO}, \mathrm{NO}_{2}, \mathrm{SO}_{2}$, ozono y material particulado $\mathrm{PM}_{10}$ y $\mathrm{PM}_{2,5}$. La evidencia científica ha demostrado una clara asociación entre la exposición a contaminantes ambientales y mayor morbilidad por asma bronquial. Al respecto hay estudios en los que se ha observado que niños asmáticos que viven cerca de carreteras tienen mayor frecuencia de asma de difícil control y en los que viven en zonas urbanas más síntomas agudos y mayor severidad de la enfermedad ${ }^{38,39}$.

\section{Manejo}

Con los 4 pasos mencionados anteriormente podemos diferenciar claramente a los pacientes con asma de difícil control de los con asma resistente a tratamiento. Al momento de evaluar a estos pacientes vemos que ambos grupos están recibiendo terapia en nivel 4 de GINA, es decir dosis altas de corticoides inhalados (Tabla 6) más un segundo controlador ya sea LABA o antileucotrienos, sin embargo, al hacer la diferenciación entre ambos grupos vemos que el manejo posterior es diferente ${ }^{5}$.

\section{Manejo del paciente con asma de dificil control}

Como hemos visto hasta ahora en los pacientes con asma de difícil control hay factores potencialmente reversibles, que al ser tratados permiten alcanzar el control de la enfermedad sin necesidad de escalar en la terapia. Incluso se ha observado que con el manejo adecuado estos pacientes logran disminuir las dosis de corticoides inhalados y mejorar su función pulmonar ${ }^{14}$. El manejo del paciente con asma de difícil control plantea un importante desafío para el especialista, requiriendo un control y seguimiento muy estrecho, idealmente con un enfrentamiento multidisciplinario, donde la educación cumple un rol central. En cada control debe evaluarse, la adherencia al tratamiento, la técnica inhalatoria y la presencia de comorbilidades. Esto último es muy importante ya que se ha visto que a pesar de un adecuado adiestramiento, siempre se observan errores en la técnica de inhalación ${ }^{38}$. Para lograr una adecuada adherencia al tratamiento es fundamental un buen programa de educación al paciente y sus cuidadores, entregando conocimientos básicos acerca de la enfermedad, enfatizando su carácter crónico y explicando a los padres lo que se pretende lograr con la terapia Es importante que la actividad educativa sea individual, a la medida del paciente, de manera de identificar si hay algún problema en particular que explique una pobre adherencia al tratamiento. Muchas veces esta labor educativa implica derribar mitos o falsas creencias que pueden incidir negativamente en la adherencia. Las intervenciones educativas aisladas son insuficientes para promover una buena adherencia, por lo que el proceso educativo debe ser continuo, idealmente en cada visita médica ${ }^{41}$. Se recomienda dar instrucciones claras y sencillas, entregadas en forma verbal y por escrito, con búsqueda de rutinas que se vinculen a la toma del medicamento (ejemplo: desayuno, lavado de dientes). Debe facilitarse el acceso a los controles médicos y en cada uno de ellos supervisar el tratamiento y reforzar conductas ${ }^{32,33}$. También es importante educar acerca de aspectos de prevención y autocuidado, de manera que el paciente sepa como evitar posibles desencadenantes de exacerbaciones y cómo actuar en caso que comience con una. $\mathrm{Al}$ respecto es importante que el paciente cuente con un plan de acción por escrito. Por otro lado, hay estudios que avalan la gran utilidad de la visita domiciliaria efectuada por enfermera, quien puede evaluar en terreno distintos aspectos relacionados con el mal control del asma como la disponibilidad del medicamento en domicilio, el ambiente en el que vive el paciente y la exposición a alérgenos y a humedad; la presencia de tabaquismo intradomiciliario y el tipo de calefacción que se utiliza. Finalmente, la

Tabla 6. Dosis altas de corticoides inhalados ${ }^{5}$

\begin{tabular}{|lcc|}
\hline Corticoide inhalado $(\mathbf{e n ~} \boldsymbol{\mu g})$ & Niños de 6 a 11 años & Niños mayores de 12 años \\
Budesonida & $>400$ & $>800$ \\
Ciclesonida & $>160$ & $>320$ \\
Fluticasona propionato & $>500$ & $>500$ \\
Fluticasona furoato & No utilizado a esta edad & 200 \\
Mometasona furoato & $>440$ & $>440$ \\
\hline
\end{tabular}


visita domiciliaria permite pesquisar problemas psicosociales y observar la dinámica familiar con respecto al cumplimiento de rutinas ${ }^{12}$. El seguimiento de estos pacientes es muy importante no sólo para ir corrigiendo en forma constante factores que incidan negativamente en el control de la enfermedad, sino porque también algunos pacientes con asma de difícil control en algún momento de su evolución pueden pasar al grupo de asmáticos resistentes a tratamiento, cuyo enfrentamiento se detalla a continuación.

\section{Manejo del paciente con asma resistente a tratamiento}

Este grupo de pacientes siempre debe ser manejado en el nivel terciario de atención, por especialista. En estos pacientes, a diferencia de los pacientes con asma de difícil control, no se encuentran factores modificables. Por lo tanto, es en este subgrupo muy seleccionado de pacientes en los que se justifica escalar en la terapia a una etapa 5 de GINA. De esta manera, al uso de altas dosis de corticoides inhalados más LABA o antileucotrienos se puede agregar un tercer controlador, ya sea una terapia biológica (omalizumab o mepolizumab) o un anticolinérgico de acción prolongada (tiotropio) y como segunda opción, si no están disponibles las anteriores, el uso de corticoides orales en dosis bajas ${ }^{5}$.

\section{Omalizumab}

Anticuerpo monoclonal humanizado anti IgE aprobado por la FDA el año 2003 y por el Instituto de Salud Pública de nuestro país el año 2009 para uso en niños mayores de 6 años. Omalizumab actúa bloqueando la IgE libre circulante, evitando que esta se una a su receptor de alta afinidad en la superficie de mastocitos y basófilos, reduciendo así la respuesta alérgica. Al haber menor cantidad de IgE libre circulante, disminuye secundariamente la expresión de receptores de alta afinidad en mastocitos, basófilos y células dendríticas, aumentado el efecto inhibitorio sobre la cascada alérgica ${ }^{42,43}$. Así, Omalizumab evita la degranulación del mastocito frente a la presencia del alérgeno, con lo que se inhibe la fase temprana de la respuesta alérgica y también se reduce indirectamente la respuesta alérgica tardía al disminuir el reclutamiento de eosinófilos. Esto último probablemente por un efecto combinado, al interferir en la detección y el procesamiento del alérgeno por parte de la célula dendrítica y al inhibir la degranulación del mastocitos, bloqueando así la liberación de citoquinas y quemoquinas encargadas de atraer células inflamatorias como eosinófilos y linfocitos ${ }^{42}$. Recientes estudios in vitro han demostrado además que omalizumab podría evitar la remodelación de la vía aérea, al disminuir el depósito de matriz extracelular y colágeno producto del bloqueo de la acción estimuladora de IgE sobre la células del músculo liso bronquial ${ }^{44}$. Desde el punto de vista clínico se ha demostrado mediante dos meta-análisis que omalizumab reduce el número de exacerbaciones y hospitalizaciones por asma bronquial, permitiendo disminuir o incluso suspender el tratamiento con corticoides orales e inhalados, acompañado además de un buen perfil de seguridad ${ }^{45,46}$. Se ha observado además que su uso reduce los días de síntomas de asma, las consultas a servicio de urgencia y el uso de medicamentos de rescate, mejorando la calidad de vida de los pacientes ${ }^{47-49}$. Por último, este anticuerpo monoclonal en niños ha demostrado ser capaz de reducir las exacerbaciones estacionales que se producen año a año en los meses de otoño producto de infecciones virales $^{50,51}$. Omalizumab es una alternativa de tratamiento para niños mayores de 6 años con asma no controlado en etapa 4 de GINA y que además presenten sensibilización al menos a un aeroalérgeno perenne en el test cutáneo o en la determinación de IgE específica y cuyos niveles de IgE total estén entre 30 a 1.500 UI. En estos pacientes la dosis y la frecuencia de administración (cada 2 o 4 semanas) dependerá del peso y del nivel de IgE total (Tablas 7 y 8). La dosis debe ajustarse sólo frente a cambios significativos en el peso. Los pacientes que tengan una concentración basal de IgE o cuyo peso corporal esté por fuera de los límites establecidos por la tabla, deben abstenerse de recibirlo. El medicamento se administra por vía subcutánea y esta disponible en dos presentaciones: vial conteniendo $150 \mathrm{mg}$ en $5 \mathrm{ml}$ y jeringa precargada con $150 \mathrm{mg}$ en 1 $\mathrm{ml}$. Se recomienda mantener el tratamiento por 12 semanas al cabo de las cuales se debe evaluar si hay o no una respuesta clínica favorable. Si la respuesta no es satisfactoria se recomienda descontinuar el tratamiento, de lo contrario se debe mantener. El tiempo total de tratamiento aun no está bien definido en la literatura, pero en general debiera ser prolongado, existiendo series con períodos de tratamiento que van entre 4 a 6 años $^{52,53}$.

\section{Mepolizumab}

Anticuerpo monoclonal humanizado antiinterleuquina 5: IL5), aprobado en noviembre de 2015 por la FDA para uso en pacientes asmáticos mayores de 12 años. En nuestro país aun no está disponible. La IL5 es una importante citoquina involucrada en la maduración y liberación de los eosinófilos desde la médula ósea a la sangre 
Tabla 7. Dosis de omalizumab (mg/dosis) administrada por inyección subcutánea cada 4 semanas

\begin{tabular}{|c|c|c|c|c|c|c|c|c|c|c|}
\hline \multirow[b]{2}{*}{$\begin{array}{l}\text { IgE basal } \\
\text { UI/ml }\end{array}$} & \multicolumn{10}{|c|}{ Peso corporal (kg) } \\
\hline & $>20-25$ & $>25-30$ & $>30-40$ & $>40-50$ & $>50-60$ & $>60-70$ & $>70-80$ & $>80-90$ & $>90-125$ & $>125-150$ \\
\hline$>30-100$ & 75 & 75 & 75 & 150 & 150 & 150 & 150 & 150 & 300 & 300 \\
\hline$>100-200$ & 150 & 150 & 150 & 300 & 300 & 300 & 300 & 300 & 450 & 600 \\
\hline$>200-300$ & 150 & 150 & 225 & 300 & 300 & 450 & 450 & 450 & 600 & \\
\hline$>300-400$ & 225 & 225 & 300 & 450 & 450 & 450 & 600 & 600 & & \\
\hline$>400-500$ & 225 & 300 & 450 & 450 & 600 & 600 & \multirow{3}{*}{\multicolumn{4}{|c|}{$\begin{array}{c}\text { Administración cada } 2 \text { semanas } \\
\text { Véase la Tabla } 8\end{array}$}} \\
\hline$>500-600$ & 300 & 300 & 450 & 600 & 600 & & & & & \\
\hline$>600-700$ & 300 & & 450 & 600 & & & & & & \\
\hline
\end{tabular}

Tabla 8.-Dosis de omalizumab (mg/dosis) administrada por inyección subcutánea cada 2 semanas

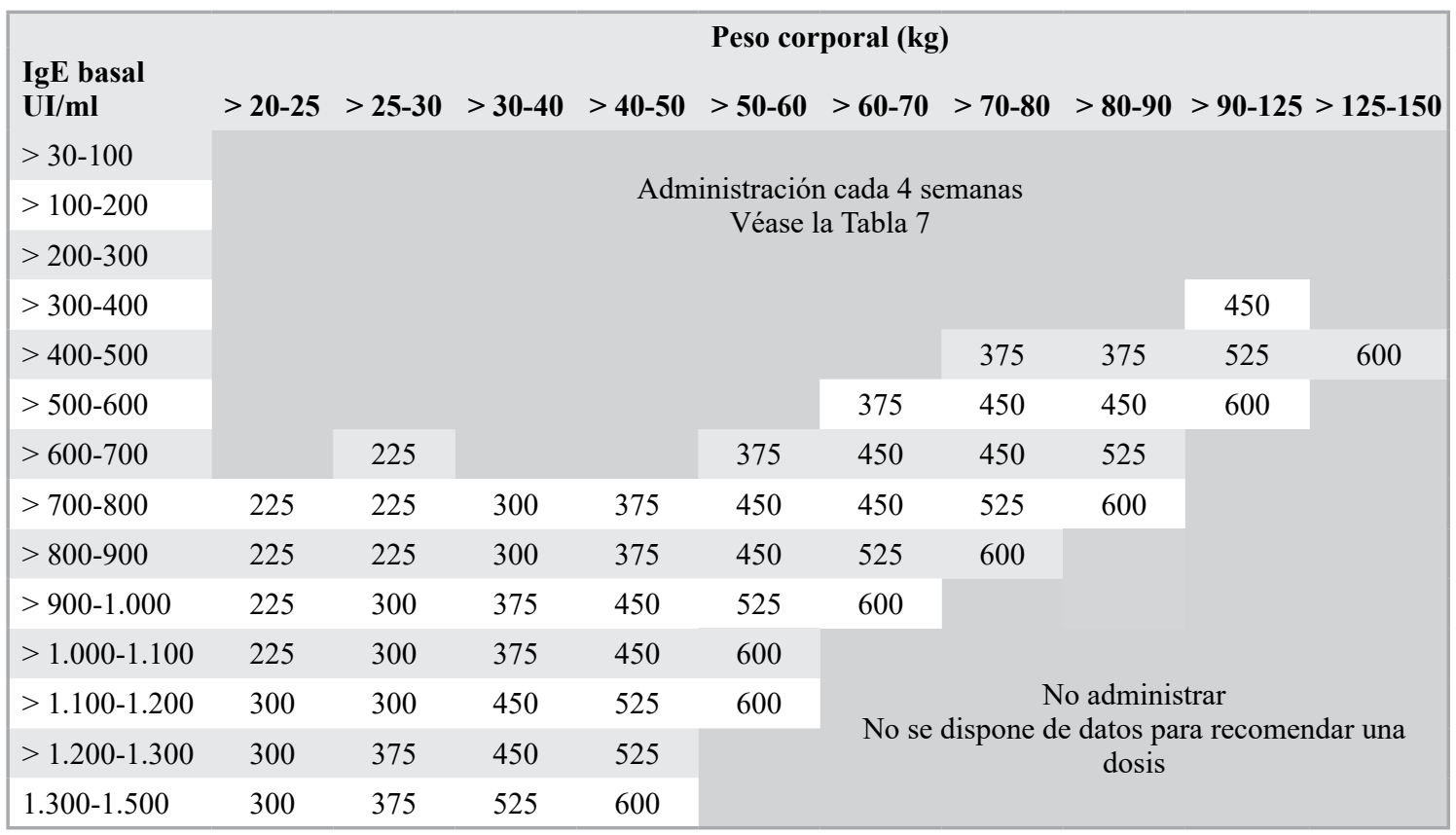

y en su reclutamiento, activación y persistencia en los tejidos ${ }^{54}$. Los eosinófilos son las células más importantes en la respuesta alérgica tardía y son las responsables de la mantención de la inflamación en la mucosa respiratoria. Mepolizumab se une con gran afinidad y especificidad a IL5, evitando que ésta interactúe con su receptor en la superficie de los eosinófilos, logrando de esta manera, disminuir la producción y la sobrevida de estas células, lo que se refleja en una reducción de su recuento en sangre y esputo ${ }^{55,56}$. Los estudios con este medicamento han demostrado una disminución significativa de las exacerbaciones, de las consultas a servicio de urgencia y de las hospitalizaciones. También se ha descrito una mejoría del control del asma y reducción del uso de corticoides orales de mantención, acompañado de un buen perfil de seguridad ${ }^{57-59}$. Este medicamento está indicado como tratamiento adicional en pacientes mayores de 12 años, con falta de control de la enfermedad a pesar de recibir tratamiento en etapa 4 de GINA y que tengan además asma de tipo eosinofílico con exacerbaciones frecuentes. Un recuento de eosinófilos en sangre mayor a 150 células $/ \mathrm{mL}$ sería un buen biomarcador para predecir una respuesta favorable a Mepolizumab y la dosis recomendada es de de $100 \mathrm{mg}$ vía subcutánea cada 4 semanas $^{54}$. Interesantemente el fenotipo que se beneficia de Mepolizumab clásicamente comienza en la edad 
adulta y si bien se presenta con eosinofilia, ésta obedece menos a un patrón alérgico a diferencia de lo que sucede en el asma de inicio precoz característico del niño ${ }^{60}$. Por último, a pesar que los estudios pivotales incluyeron niños mayores de 12 años, su número era muy escaso con respecto a la muestra total, por lo que se requiere cautela al momento de decidir indicar este tipo de terapia a niños entre 12 y 17 años.

\section{Tioptropio}

La liberación de acetilcolina desde los nervios parasimpáticos lleva a la contracción de la musculatura lisa bronquial, secreción de mucus y vasodilatación. Esto se produce por estimulación de los receptores muscarínicos M3 a nivel pulmonar. La acetilcolina induce además la liberación de mediadores pro-inflamatorios desde las células del epitelio respiratorio bronquial y desde células del sistema inmune incluyendo macrófagos, mastocitos, monocitos, granulocitos, neutrófilos y eosinófilos. Los antagonistas muscarínicos compiten con acetilcolina por su receptor, así tienen efecto broncodilatador y antiinflamatorio $^{61}$. Tiotropio es un agente antimuscarínico de vida media larga (LAMA) y ha sido utilizado para el tratamiento de la enfermedad pulmonar obstructiva crónica (EPOC) desde hace más de 10 años. Recientemente ha sido aprobado por la FDA para el tratamiento del asma en pacientes mayores de 12 años, en nivel 4 o 5 de GINA. Los estudios disponibles demuestran que su uso mejora la función pulmonar y el control del asma. Reduce las exacerbaciones y mejora la calidad de vida tanto en niños como en adultos, con un buen perfil de seguridad ${ }^{62,63}$. Este medicamento se administra por vía inhalada mediante un dispositivo llamado Respimat el cual está libre de propelentes. Produce una nube de aerosol a partir de una solución acuosa, generando partículas de 1 a $5 \mu \mathrm{m}$ de diámetro, con un depósito pulmonar cercano al $40 \%{ }^{64}$. Cada inhalación entrega $2,5 \mu \mathrm{g}$ de tiotropio, siendo la dosis recomendada 2 inhalaciones una vez al día ( $5 \mu \mathrm{g} /$ día). Es importante señalar que los estudios randomizados con este medicamento en adolescentes son escasos y con un número de pacientes relativamente pequeño, por lo que se requieren ensayos clínicos con mayor número de participantes y de mayor duración para poder establecer con mayor grado de evidencia la eficacia y seguridad de tiotropio en esta población.

\section{Corticoides orales}

De acuerdo a las guías GINA el uso de corticoides orales en dosis bajas podría ser una alter- nativa de segunda línea para pacientes con asma severo resistente a tratamiento, en los cuales no se logre el control con tratamiento en etapa 4 de GINA y en los que no haya posibilidad de utilizar otras terapias ${ }^{5}$. Sin embargo, hay que tratar de evitar a toda costa el uso de corticoides orales en forma permanente, especialmente en la población pediátrica, debido a sus múltiples efectos adversos, tales como osteoporosis, cataratas, insuficiencia adrenal y problemas de crecimiento ${ }^{65}$. En la literatura no hay información clara acerca de cuál sería la dosis de corticoides orales a utilizar ni por cuánto tiempo mantenerlos, como tampoco se detalla la forma más adecuada de disminuirlos. Basándonos en la recomendación de expertos una dosis inicial podría ser $0,5 \mathrm{mg} / \mathrm{kg} /$ día con un tope diario de 7,5 $\mathrm{mg}$. Se recomienda realizar una prueba terapéutica por 14 días. Si al cabo de de este tiempo no se nota una mejoría clínica significativa el tratamiento debería ser suspendido. En caso contrario la dosis se debe disminuir hasta llegar a la mínima posible que sea capaz de mantener el control ${ }^{65}$. El tratamiento puede ser administrado día por medio. Los pacientes con asma que estén en tratamiento con corticoides orales permanentes deberían tener control mensual de peso, talla, presión arterial y glicemia. Adicionalmente deberían tener evaluación oftalmológica y endocrinológica para descartar la presencia de cataratas y osteoporosis.

\section{Terapias en desarrollo}

Actualmente están en desarrollo otras terapias biológicas aun no aprobados por la FDA. La IL-4 y la IL-13 constituyen una importante diana terapéutica en asma, ya que tienen un papel muy relevante en la respuesta de los linfocitos Th2 y en el cambio de isotipo de los linfocitos B para la síntesis de IgE. Es así como se están ensayando las siguientes terapias:

Lebrikizumab: Anticuerpo monoclonal humanizado anti IL-13. Su administración es por vía subcutánea. Su uso en adultos ha demostrado una mejoría de la función pulmonar y reducción de las exacerbaciones en un $60 \%$, pero sólo en un subgrupo de pacientes que tienen elevación de un biomarcador en sangre llamado periostina ${ }^{66,67}$.

Pitakinra: Es un análogo de IL-4 que bloquea la subunidad alfa del receptor de esta interleuquina. La subunidad alfa del receptor es común para IL-4 e IL-13. De esta manera se inhibe simultáneamente la acción de IL-4 e IL-13 ${ }^{68}$. Existe una presentación subcutánea y una inhalada, siendo de mayor eficacia la segunda. En estudios de fase II se demostró que su uso reduce la inflamación de la vía aérea y mejora la función pulmonar ${ }^{69}$. 
Dupilumab: Anticuerpo monoclonal recombinante completamente humano, que actúa bloqueando la subunidad alfa del receptor de IL-4, con lo que se inhibe la acción de IL-4 e IL-13. Se administra por vía subcutánea. Su uso en adultos ha demostrado una reducción significativa del número de exacerbaciones acompañado de una mejoría de la función pulmonar y del grado de control del asma ${ }^{70,71}$.

\section{Bibliografía}

1.- FISCHER G B, CAMARGOS P A, MOCELIN H T. The burden of asthma in children: a Latin American perspective. Paed Resp Rev 2005; 6: 8-13.

2.- GODARD P, CHANEZ P, SIRAUDIN L, NICOLOYANNIS N, DURU G. Costs of asthma are correlated with severity: a 1-year prospective study. Eur Respir J 2002; 19: 61-7.

3.- GUILBERT T W, BACHARIER L B, FITZPATRICK A M. Severe asthma in children. J Allergy Clin Immunol Pract 2014; 2 : 489-500.

4.- BOUSQUET J, MANTZOURANIS E, CRUZ A A, AIT-KHALED N, BAENNA-CAGNANI C E, BLEECKER E R, et al. Uniform definition of asthma severity, control, and exacerbations: document presented for the World Health Organization Consultation on Severe Asthma. J Allergy Clin Immunol 2010; 126: 926-38.

5.- GINA: Update 2016 Disponible en: www.ginasthma. com

6.- CHUNG K F, WENZEL S E, BROZEK J L, BUSH A, CASTRO M, STERK P J, et al. International ERS/ATS guidelines on definition, evaluation and treatment of severe asthma. Eur Respir J 2014; 43: 343-73.

7.- BUSH A, ZAR H J. WHO universal definition of severe asthma. Curr Opin Allergy Clin Immunol 2011; 11: 11521.

8.- AMERICAN THORACIC SOCIETY. Proceedings of the ATS workshop on refractory asthma: current understanding, recommendations, and unanswered questions. Am J Respir Crit Care Med 2000; 162: 2341-51.

9.- BEL E H, SOUSA A, FLEMING L, BUSH A, CHUNG K F, VERSNEL J, et al. Diagnosis and definition of severe refractory asthma: an international consensus statement from the Innovative Medicine Initiative (IMI). Thorax 2011; 66: 910-7.

10.- HEDLIN G, BUSH A, LODRUP CARLSEN K, WENNERGREN G, DE BENEDICTIS F M, MELEN E, et al. Problematic severe asthma in children, not one problem but many: a GA2LEN initiative. Eur Respir J 2010; 36: 196-201.

11.- BUSH A, SAGLANI S. Management of severe asthma in children. Lancet 2010; 376: 814-25.

12.- BRACKEN M, FLEMING L, HALL P, VAN STIPHOUT N, BOSSLEY C, BIGGART E, et al. The importance of nurse-led home visits in the assessments of children with problematic asthma. Arch Dis Child 2009; 94: 780-4.

13.- KONRADSEN J R, NORDLUND B, LIDEGRAN M, PEDROLETTI C, GRONLUND H, VAN HAGE M, et al. Problematic severe asthma: a proposed approach to identifying children who are severely resistant to therapy. Pediatr Allergy Immunol 2011; 22: 9-18.

14.- SHARPLES J, GUPTA A, FLEMING L, BOSSLEY C J, BRACKEN-KING M, HALL P, et al. Long term effectiveness of a staged assessment for paediatric problematic severe asthma. Eur Respir J 2012; 40: 264-7.

15.- BOZZETTO S, CARRARO S, ZANCONATO S, BARALDI E. Severe asthma in childhood: diagnostic and management challenge. Curr Opin Pulm Med 2015, 21 : 16-21.

16.- FOKKENS W, LUND V J, MULLOL J, BACHERT C, ALOBID I, BAROODY F, et al. EPOS 2012: European position paper on rhinosinusitis and nasal polyps. Rhinology 2012; 50: 1- 12.

17.- PIJNENBURG M W, BARALDI E, BRAND P L, CARLSEN K H, EBER E, FRISCHER T, et al. Monitoring Asthma in Children. Eur Respir J 2015; 45: 906-25.

18.- BROZEK J L, BOUSQUET J, BAENA-CAGNANI C E, BONINI S, CANONICA G W, CASALE T B, et al. Allergic Rhinitis and its impact in Asthma (ARIA) guidelines: 2010 revision. J Allergy Clin Inmunol 2010; 126: 466-76.

19.- TOLIA V, VANDENPLAS Y. Systematic review: the extra-oesophageal symptoms of gastro-oesophageal reflux disease in children. Aliment Pharmacol Ther 2009; 29: 258-72.

20.- CHAN W W, CHIOU E, OBSTEIN K L, TIGNOR A S, WHITLOCK T L. The efficacy of proton pump inhibitors for the treatment of asthma in adults: a metaanalysis. Arch Intern Med 2011; 171: 620-9.

21.- HOLGUIN F, BLEECKER E R, BUSSE W W, CALHOUN W J, CASTRO M, ERZURUM S C, et al. Obesity and asthma: an association modified by age of asthma onset. J Allergy Clin Immunol 2011; 127: 148693.

22.- SCOTT H A, GIBSON P G, GARG M L, PRETTO J J, MORGAN P J, CALLISTER R, et al. Dietary restriction and exercise improve airway inflammation and clinical outcomes in overcomes in overweight and obese asthma: a randomized trial. Clin Exp Allergy 2013; 43 : 36-49.

23.- MORGAN M D. Dysfunctional breathing in asthma: is it common, identifiable and correctable? Thorax 2002; 57 (Suppl 2): ii31-5.

24.- BROUWER A F, ROORDA R J, BRAND P L. Treatment of asthma in children: more than merely “puffing”. Ned Tijdschr Geneeskd 2004; 148: 865-8.

25.- DE GROOT E P, DUIVERMAN E J, BRAND P L. Comorbidities of asthma during childhood possibly important, yet poorly studied. Eur Respir J 2010; 36: 671-8. 
26.- SIMPSON A B, GLUTTING J, YOUSEF E. Food allergy and asthma morbidity in children. Pediatr Pulmonol 2007; 42: 489-95.

27.- KATON W J, RICHARDSON L, RUSSO J, LOZANO P, MCCAULEY E. Quality of mental health care for youth with asthma and comorbid anxiety and depression. Med Care 2006; 44: 1064-72.

28.- JENTZSCH N S, CAMARGOS P, SARINHO E S, BOUSQUET J. Adherence rate to beclomethasone dipropionate and the level of asthma control. Respir Med 2012; 106: 338-43.

29.- MORTON R W, EVERARD M L, ELPHIC H E. Adherence in childhood asthma: the elephant in the room. Arch Dis Child 2014; 99: 949-53.

30.- KLOK T, KAPTEIN A A, DUIVERMANN E J, BRAND P L. It's the adherence, stupid (that determines asthma control in preschool children). Eur Respir J 2014; 43: 783-91.

31.- NAVARRO M, PÉREZ G, ROMERO M. Causas de asma de control difícil (ACD). Factores que pueden agravar el asma. An Pediatr (Barc) 2005; 62 (Supl 1): $35-40$.

32.- KLOK T, KAPTEIN A A, BRAND P L. Non-adherence in children with asthma reviewed: The need for improvement of asthma care and medical education. Pediatr Allergy Immunol 2015; 26: 197-205.

33.- PAPADOPOULOS N G, ARAKAWA H, CARLSEN K H, CUSTOVIC A, GERN J, LEMANSKE R, et al. International consensus on (ICON) pediatric asthma. Allergy 2012; 67: 976-97.

34.- CONSENSO SEPAR-ALAT sobre terapia inhalada. Arch Bronconeumol 2013; 49 (Supl 1): 2-14.

35.- LAUBE B L, JANSSENS H M, JONGH F H C, DEVADASON S G, DHAND R, DIOT P, et al. What the pulmonary specialist should know about the new inhalation therapies. Eur Respir J 2011; 37: 1308-14.

36.- RUEDAS S. Sistemas de inhalación en el asma infantil. An Pediatr Contin 2012; 10: 148-57.

37.- KANCHONGKITTIPHON W, MENDELL M J, GAFFIN J M, WANG G, PHIPATANAKUL W. Indoor environmental exposures and exacerbation of asthma: an update to the 2000 review by the Institute of Medicine. Environ Health Perspect 2015; 123: 6-20.

38.- SAGLANI S, FLEMING L. How to manage a child with difficult asthma? Expert Rev Respir Med 2016; 2 : 1-7.

39.- MILLIGAN K L, MATSUI E, SHARMA H. Asthma in Urban Children: Epidemiology, Environmental Risk Factors, and the Public Health Domain. Curr Allergy Asthma Rep 2016; 16: 33. doi 10.1007/s11882-0160609-6

40.- CENTRO NACIONAL DEL MEDIO AMBIENTE. CENMA. Evaluación de impacto atmosférico de sistemas de calefacción domiciliaria. Junio 2011. Disponible en: http://studylib.es/doc/691667/descarga-glp-chile\#. V1RAc1jlHEM.gmail.
41.- DEAN A J, WALTERS J, HALL A. A systematic review of interventions to enhance medication adherence in children and adolescents with chronic illness. Arch Dis Child 2010; 95: 717-23.

42.- HOLGATE S T. New strategies with anti-IgE in allergic diseases. World Allergy Org J. 2014; 7: 17-22.

43.- LICARI A, MARSEGLIA A, CAIMMI S, CASTAGNOLI R, FOIADELLI T, BARBERI S, et al. Omalizumab in children. Paediatr Drugs 2014; 16: 491-502.

44.- ROTH M, ZHAO F, ZHONG J, LARDINOIS D, TAMM M. Serum IgE induced airway smooth muscle cell remodeling is independent of allergens and is prevented by omalizumab. PLoS One 2015; 10: e0136549.

45.- NORMANSELL R, WALKER S, MILAN S J, WALTERS E H, NAIR P. Omalizumab for asthma in adults and children. Cochrane Database Syst Rev 2014; (1): CD003559.

46.- RODRIGO G J, NEFFEN H, CASTRO-RODRÍGUEZ $\mathrm{J}$ A. Efficacy and safety of subcutaneous omalizumab vs placebo as add-on therapy to corticosteroids for children and adults with asthma: a systematic review. Chest 2011; 139: 28-35.

47.- BRAUNSTAHL G J, CHEN C W, MAYKUT R, GEORGIOU P, PEACHEY G, BRUCE J. The experience registry: the 'real-world' effectiveness of omalizumab in allergic asthma. Respir Med 2013; 107: 1141-51.

48.- HUMBERT M, BUSSE W, HANANIA N A, LOWE P J, CANVIN J, ERPENBECK V J. Omalizumab in asthma: an update on recent developments. J Allergy Clin Immunol Pract 2014; 2: 525-36.

49.- HUMBERT M, BEASLEY R, AYRES J, SLAVIN R, HÉBERT J, BOUSQUET J. Benefits of omalizumab as add-on therapy in patients with severe persistent asthma who are inadequately controlled despite best available therapy (GINA 2002 step 4 treatment): INNOVATE. Allergy 2005; 60: 309-16.

50.- BUSSE W W, MORGAN W J, GERGEN P J, MITCHELL H E, GERN J E, LIU A H, et al. Randomized trial of omalizumab (anti-IgE) for asthma in inner-city children. N Engl J Med 2011; 364: 1005-15.

51.- TEACH S J, GILL M A, TOGIAS A, SORKNESS C A, ARBES S J JR, CALATRONI A. Preseasonal treatment with either omalizumab or an inhaled corticosteroid boost to prevent fall asthma exacerbations. J Allergy Clin Immunol 2015; 136: 1476-85.

52.- LAI T, WANG S, XU Z, ZHANG C, ZHAO Y, HU $Y$. Long-term efficacy and safety of omalizumab in patients with persistent uncontrolled allergic asthma: a systematic review and meta-analysis. Sci Rep 2015; 5: 8191.

53.- NOPP A, JOHANSSON S G, ADÉDOYIN J, ANKERST J, PALMQVIST M, OMAN H. After 6 years with Xolair; a 3-year withdrawal follow-up. Allergy 2010; 65: 56-60.

54.- WALSH G M. Severe eosinophilic asthma and mepolizumab. Lancet Respir Med 2016; 4: 528-9. 
55.- DEEKS E D. Mepolizumab: A Review in Eosinophilic Asthma. BioDrugs 2016 Jun 16 (Epub ahead of print).

56.- HALDAR P, BRIGHTLING C E, HARGADON B, GUPTA S, MONTEIRO W, SOUSA S. Mepolizumab and exacerbations of refractory eosinophilic asthma. N Engl J Med 2009; 360: 973-84.

57.- PAVORD I D, KORN S, HOWARTH P, BLEECKER E R, BUHL R, KEENE O N, et al. Mepolizumab for severe eosinophilic asthma (DREAM): a multicentre, double-blind, placebo-controlled trial. Lancet 2012; 380: 651-9.

58.- ORTEGA H G, LIU M C, PAVORD I D, BRUSSELLE G G, FITZGERALD J M, CHETTA A, et al. Mepolizumab treatment in patients with severe eosinophilic asthma. N Engl J Med 2014; 371: 1198-207.

59.- BEL E H, WENZEL S E, THOMPSON P J, PRAZMA C M, KEENE O N, YANCEY S W, et al. Oral glucocorticoid-sparing effect of mepolizumab in eosinophilic asthma. N Engl J Med 2014; 371: 1189-97.

60.- BLAKE K, RAISSY H. Mepolizumab: A new class of treatment for adolescents with severe persistent asthma. Pediatr Allergy Immunol Pulmonol 2016; 29: 49-52.

61.- BUSSE W W, DAHL R, JENKINS C, CRUZ A A. Long-acting muscarinic antagonists: a potential add-on therapy in the treatment of asthma? Eur Respir Rev 2016; 25: 54-64.

62.- VOGELBERG C. Emerging role of long-acting anticholinergics in children with asthma. Curr Opin Pulm Med 2016; 22: 74-9.

63.- RODRIGO G J, CASTRO-RODRÍGUEZ J A. Tiotropium for the treatment of adolescents with moderate to severe symptomatic asthma: a systematic review with meta-analysis. Ann Allergy Asthma Immunol 2015; 115: 211-6.
64.- LYSENG-WILLIAMSON K A, KEATING G M. Tiotropium Respimat Soft Mist inhaler: a guide to its use in chronic obstructive pulmonary disease (COPD) in the EU. Drugs and Therapy Perspectives 2015; 31: 39-44.

65.- BUSH A, PEDERSEN S, HEDLIN G, BARALDI E, BARBATO A, DE BENEDICTIS F, et al. Pharmacological treatment of severe, therapy-resistant asthma in children: what can we learn from where? Eur Respir J 2011; 38: 947-58.

66.- CORREN J, LEMANSKE R F, HANANIA N A, KORENBLAT P E, PARSEY M V, ARRON J R, et al. Lebrikizumab treatment in adults with asthma. N Engl J Med 2011; 365: 1088-98.

67.- MASELLI D J, KEYT H, ROGERS L. Profile of lebrikizumab and its potential in the treatment of asthma. $\mathrm{J}$ Asthma Allergy 2015; 8: 87-92.

68.- SLAGER R E, OTULANA B A, HAWKINS G A, YEN Y P, PETERS S P, WENZEL S E, et al. IL-4 receptor polymorphisms predict reduction in asthma exacerbations during response to an anti-IL-4 receptor $\alpha$ antagonist. J Allergy Clin Immunol 2012; 130: 516-22.

69.- ANTONIU S A, COJOCARU I. Pitrakinra for asthma. Expert Opin Biol Ther 2010; 10: 1609-15.

70.- WENZEL S, FORD L, PEARLMAN D, SPECTOR S, SHER L, SKOBIERANDA F, et al. Dupilumab in persistent asthma with elevated eosinophil levels. N Engl J Med 2013; 368: 2455-66.

71.- WENZEL S, CASTRO M, CORREN J, MASPERO J, WANG L, ZHANG B, et al. Dupilumab efficacy and safety in adults with uncontrolled persistent asthma despite use of medium-to-high-dose inhaled corticosteroids plus a long-acting $\beta 2$ agonist: a randomised doubleblind placebo-controlled pivotal phase $2 \mathrm{~b}$ dose-ranging trial. Lancet 2016; 388 (10039): 31-44.
Correspondencia a:

Dra. Ana María Herrera Gana

Clínica Santa María

Santiago de Chile

Email: amherreragana@gmail.com 